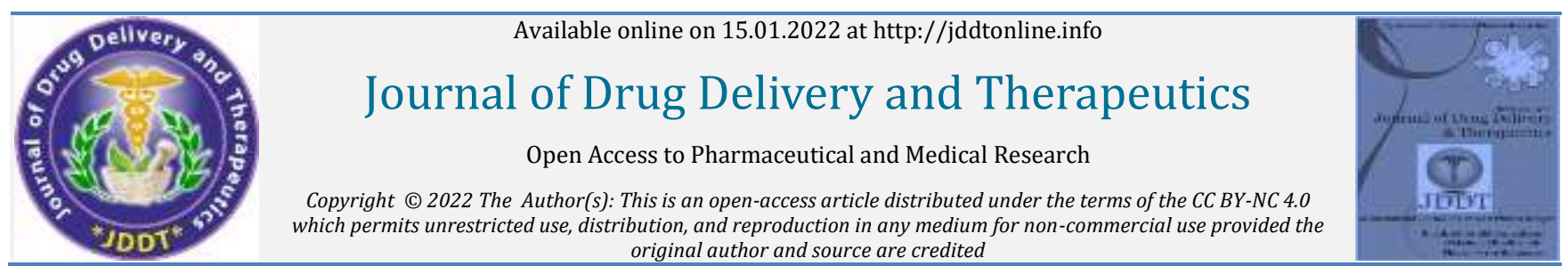
original author and source are credited

\title{
Assessment of Drug Utilization Pattern and Rational Use of Antihypertensive Therapy in Geriatric Patients: A Prospective Observational Study
}

\author{
AHM Viswanatha Swamy ${ }^{1}$ (1), Mahendra Kumar R ${ }^{1}$, Sanat B. Nyamagoud ${ }^{1 *}$ (i), Jaseen James ${ }^{2}$ (ii), \\ Jesmitha James 2(19), Manna Siby 2(ii), Anit Mathews 2(ii), \\ ${ }^{1}$ Department of Pharmacy Practice, KLE College of Pharmacy Hubballi, Dharwad, India- 580031 \\ 2 Interns, Department of Pharmacy Practice, KLE College of Pharmacy Hubballi, Dharwad, India- 580031
}

Article Info:

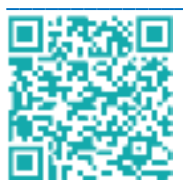

Article History:

Received 13 November 2021 Reviewed 16 December 2021 Accepted 21 December 2021

Published 15 January 2022

\section{Cite this article as:}

Viswanatha Swamy AHM, R MK, Nyamagoud SB, James J James J, Siby M, Mathews A, Assessment of Drug Utilization Pattern and Rational Use of Antihypertensive Therapy in Geriatric Patients: A Prospective Observational Stud, Journal of Drug Delivery and Therapeutics. 2022; 12(1):72-77

DOI: http://dx.doi.org/10.22270/jddt.v12i1.5256

\section{*Address for Correspondence:}

Dr. Sanath. B. Nyamagoud, Assistant Professor, Department of Pharmacy Practice, KLE College of Pharmacy Hubballi, India

ORCID ID: https://orcid.org/0000-0002-5111-7685

\section{Abstract}

Background: Hypertension is one of the most frequently occurring noncommunicable diseases, and it causes a variety of cardiovascular complications and mortality. Diagnosis and treatment in elderly patients are very difficult due to their changes in physiology and physiological responses to the therapy. Drug utilization studies are plausible instrument in the evaluation in health care practice. Objective: To assess drug utilization pattern and rational use of antihypertensive therapy in geriatric patients. Materials and methods: This is a prospective observational study that took place in 200 inpatients over the course of six months. The study includes subjects with age group of 65 years and above with all comorbidities. The collected data was statistically evaluated by appropriate descriptive statistical analysis. Here the prescription pattern and rationality were analysed. Results: In this study out of 200 patients, $53 \%$ of prescriptions contain combination therapy and remaining $47 \%$ contains mono therapy. Most commonly prescribed class as mono therapy was Calcium channel blockers 79. 00\% (Amlodipine). Among two drug regimen Calcium channel blockers + Diuretics and the three-drug regimen Calcium channel blockers + Angiotensin Receptor Blocker + Diuretic combination are generally prescribed. Here the overall rate of adherence to JNC 8 is 95\%. Conclusion: In this study by analysing prescription pattern, we concluded that most commonly prescribed drug class was Calcium channel blockers (Amlodipine). The present study results demonstrate that, most of the physicians are adherent to the JNC 8 recommendations.

Keywords: Antihypertensive drugs, Adherence to JNC 8, Rational use, Prescription patterns.

\section{INTRODUCTION}

Hypertension is a paramount independent stake factor for a variety of diseases. It's linked to a lot of morbidity and mortality in diverse populations, which has a large economic impact. Antihypertensive agents are one of the widely used therapeutic agents due to increased prevalence of Hypertension and their heterogenic therapeutic efficacy. Most of the randomized controlled trials have concluded that early management of high blood pressure can significantly reduce the risk of coronary artery disease, end stage renal disease, CHF, stroke and gross morbidity. The guidelines by the JNC are still considered as gold standard. Each updated version of JNC guidelines concentrates on drug treatment threshold and drug choices. Geriatric patients are more vulnerable to drug related problems because of their agerelated changes in physical, physiological, and psychological aspects. A number of risk factors are assumed to increase the risk of drug related problem in geriatrics, such as over use of drugs, inappropriate use, medication errors, medication nonadherence etc. The literature reviews give confidence that the outcome of the study aspect definitely gives a positive impact on society and Healthcare professionals to take necessary steps to prevent the drug related problems in the geriatric population. This study primarily focuses on the assessment of drug utilization patterns and rationally of prescribing antihypertensive agents in elder patients with co morbid conditions. It is very essential to study and rise a question on how the drugs are prescribed and what is the rationale behind the prescription.

\section{MATERIALS AND METHODS}

Study site: Vivekananda General Hospital, Deshpande Nagar Hubballi.

Study design: This is a Prospective observational study.

Study period: This study was conducted for a period of 6 months.

Study subjects: This study included the in-patients admitted in Vivekananda hospital Hubbali.

Ethical Approval: Ethical clearance for this study was obtained from institutional ethical committee. (Ref. No. KLECOPH/IEC/2020-21/05) 
Study criteria: The study includes geriatric population $(\geq 65$ years), patients of both the gender and patients with all comorbid conditions attending inpatient department. The study excludes patients who are attending outpatient department, patients with the age group of less than 65 years, patients with psychiatric disorders, patients admitted in ICU, patients with incomplete medical records and patients who are not willing to participate in the study.

Data collection: The data is collected with a suitable patient data collection form.

Statistical method: The data is statistically tested with the help of Descriptive analysis.

\section{RESULTS}

\section{Distribution of demographic data of subjects}

Out of 200 study populations $54.00 \%$ were females $(n=108)$ and $46.00 \%$ were males $(n=92)$. The mean age of the study participants was found to be $69.7 \pm 7.66$. The results shown that subjects of age group between 65 to $70(74 \%)$ were most affected and $33 \%(n=66)$ subjects were smokers and $36.50 \%(n=73)$ were alcoholics. Among study population, $41.00 \%(\mathrm{n}=82)$ subjects were under APL and $59.00 \%$ $(\mathrm{n}=118)$ were BPL and $34.50 \%(\mathrm{n}=69)$ were literate and $65.50 \%(n=131)$ were Illiterate $\{$ Table No.1\}.

In this study it was found that the subjects were diagnosed with hypertension mostly since 5 to 10 years (35\%) \{Fig.1\}.
In the study it was found that out of the selected study population $36 \%(n=138)$ were diagnosed with type 2 DM followed by $15 \%(n=57)$ were diagnosed with CNS diseases.

\begin{tabular}{|l|l|}
\hline \multicolumn{2}{|l|}{ Table No.1 Distribution of Demographic data } \\
\hline Demographic Data & Percentage (N) \\
\hline Age & $74(148)$ \\
\hline $65-70$ & $12.5(25)$ \\
\hline $71-75$ & $7(14)$ \\
\hline $76-80$ & $6(12)$ \\
\hline $81-85$ & $0.5(1)$ \\
\hline$>85$ & $46(92)$ \\
\hline Gender & $54(108)$ \\
\hline Male & \\
\hline Female & $33(66)$ \\
\hline Personal History & $67(134)$ \\
\hline Smoking & $36(73)$ \\
\hline Non-smoking & $64(127)$ \\
\hline Alcoholic & $34(69)$ \\
\hline Non-alcoholic & $66(131)$ \\
\hline Educational Status & $41(82)$ \\
\hline Literate & $59(118)$ \\
\hline Illiterate & \\
\hline Economic Status & \\
\hline APL & \\
\hline BPL &
\end{tabular}

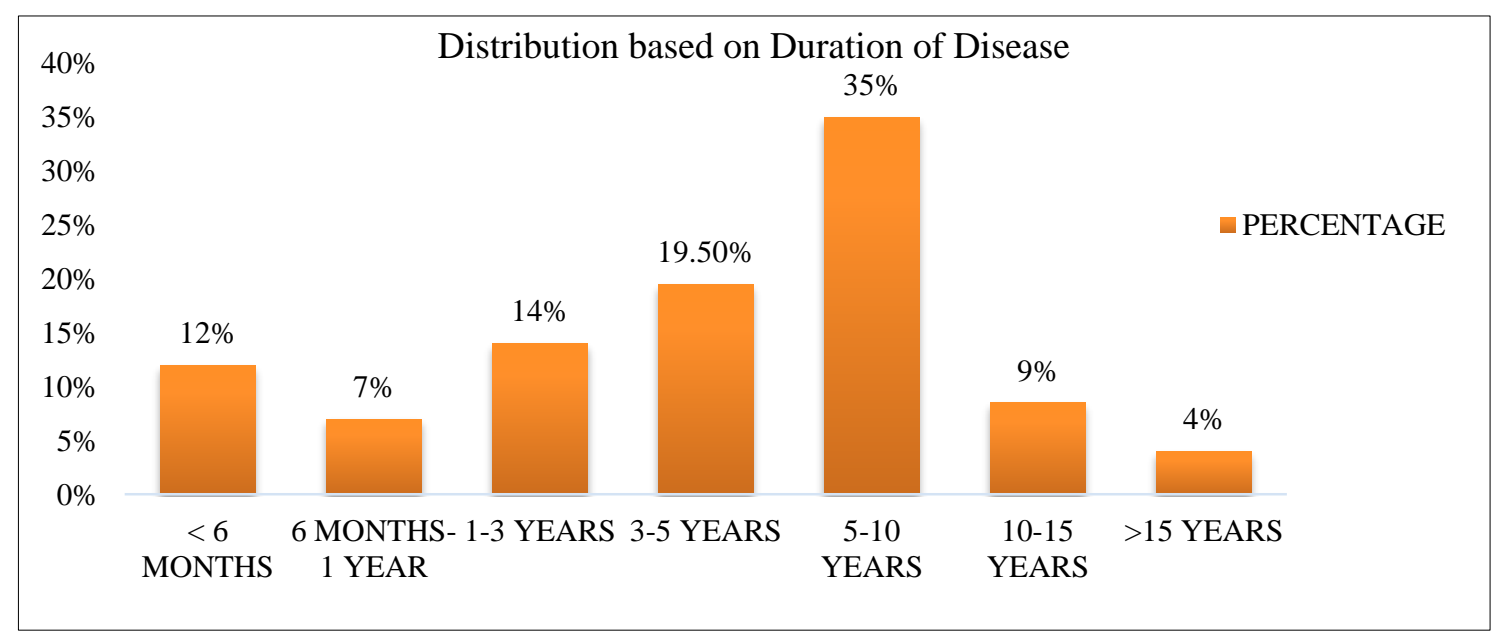

Figure 1: Distribution of subjects based on duration of disease.

\section{Prescription pattern analysis}

In the study it was found that the antihypertensive treatment given to the hypertensive patients were of 2 types, monotherapy $47.00 \% \quad(n=94)$ and combination therapy $53.00 \%(n=106)$. In this study out of 23 combination therapy with FDC, $91.30 \%(n=21)$ were combination therapy with one FDC and $8.70 \%(n=2)$ were combination therapy with two FDC $\{$ Table No.2\}.

\begin{tabular}{|l|l|}
\hline \multicolumn{2}{|l|}{ Table No.2 Distribution of subjects based on type of therapy, Combination therapy with FDCs } \\
\hline Type of Therapy & Percentage(N) \\
\hline Monotherapy & $47(94)$ \\
\hline Combination therapy & $53(106)$ \\
\hline Combination Therapy with FDCs & $91.3(21)$ \\
\hline Combination therapy with 1 FDC & $8.7(2)$ \\
\hline Combination therapy with 2 FDCs &
\end{tabular}


AHM Viswanatha Swamy et al

Among 93 monotherapies, CCBs were most commonly preferred class $79 \%(\mathrm{n}=74)$. By analysing distribution of two drug combination therapy, out of 61, CCB + Loop Diuretic was the most routinely prescribed combination therapy $44.27 \% \quad(n=27) \quad$ Table No.3\}. Out of 34 three drug combination therapy, the most commonly prescribed combination was CCB+ Diuretic+ ARB 15\% (n=5). By evaluating the distribution of 4 and 5 drug combination therapy, it was found that, 4 drug combination CCB+ Alpha Beta Blocker + ARB +Diuretic 18\%(n=2) and 5 drug

\begin{tabular}{|c|c|}
\hline \multicolumn{2}{|c|}{$\begin{array}{l}\text { Table 3: Distribution of Monotherapy and two drug } \\
\text { combination therapy. }\end{array}$} \\
\hline Distribution of Monotherapy & $\begin{array}{l}\text { Percentage } \\
\text { (N) }\end{array}$ \\
\hline Thiazide diuretics & $1.06(1)$ \\
\hline ACE Inhibitors & $3.19(3)$ \\
\hline ARBs & $7.44(7)$ \\
\hline Beta Blockers & $8.51(8)$ \\
\hline Alpha and Beta Blocker & $1.06(1)$ \\
\hline CCB & $79(74)$ \\
\hline \multicolumn{2}{|l|}{ Distribution of 2 Drug Therapy } \\
\hline ACE Inhibitors+ Beta Blocker & $1.64(1)$ \\
\hline ACE Inhibitors +Loop Diuretic & $4.9(3)$ \\
\hline ARBs +Beta Blocker & $4.92(3)$ \\
\hline ARBs+ Loop Diuretic & $3.28(2)$ \\
\hline ARBs+ Thiazide Diuretic & $3.28(2)$ \\
\hline CCB + ACE Inhibitors & $1.64(1)$ \\
\hline CCB +Alpha Beta Blocker & $6.56(4)$ \\
\hline CCB+ Alpha Agonist & $3.28(2)$ \\
\hline CCB+ARBs & $6.56(4)$ \\
\hline CCB+ Beta Blocker & $11.48(7)$ \\
\hline CCB+ Loop Diuretic & $44.27(27)$ \\
\hline Loop Diuretic + Loop Diuretic & $1.64(1)$ \\
\hline $\begin{array}{l}\text { Loop Diuretic+ Potassium Sparing } \\
\text { Diuretic }\end{array}$ & $1.64(1)$ \\
\hline Loop Diuretic +Beta Blocker & $3.28(2)$ \\
\hline $\begin{array}{l}\text { Potassium Sparing Diuretic+ Beta } \\
\text { Blocker }\end{array}$ & $1.64(1)$ \\
\hline
\end{tabular}

Journal of Drug Delivery \& Therapeutics. 2022; 12(1):72-77 combination CCB+ Diuretic +Alpha Beta Blocker +Diuretic +Alpha Agonist 18\% ( $\mathrm{n}=2)$ were prescribed most \{Table No.4\}. In the study Amlodipine was prescribed more commonly (72\%) compared to other drugs. Out of 26 prescribed antihypertensive agents, 24 drugs are given in the form of tablet. Amlodipine, belongs to the class of CCB, is the most commonly prescribed drug in the study population 49.48\% (n=143). Only 2 antihypertensive drugs were prescribed as injection/infusion form. In that, Furosemide was the most commonly prescribed drug $82.61 \%(n=57)$.
Table 4: Distribution of 3 drug therapy, Distribution of $4 \& 5$ drug therapy.

\begin{tabular}{|l|l|}
\hline Distribution of 3 Drug Therapy & $\begin{array}{l}\text { Percentage } \\
(\mathrm{N})\end{array}$ \\
\hline Alpha Agonist + ARBs + Beta Blocker & $3(1)$ \\
\hline Alpha Beta Blocker +ARBs+ CCB & $3(1)$ \\
\hline CCB+ ACE Inhibitor +Beta Blocker & $2.94(1)$ \\
\hline CCB+ Alpha Beta Blocker +ACE Inhibitor & $6(2)$ \\
\hline CCB+ Diuretics +ACE Inhibitor & $2.94(1)$ \\
\hline CCB+ Diuretics + Alpha Agonist & $3(1)$ \\
\hline CCB+ Diuretics +Alpha Beta Blocker & $6(2)$ \\
\hline CCB+ Diuretics+ ARBs & $15(5)$ \\
\hline CCB+ Diuretics+ Beta Blocker & $12(4)$ \\
\hline CCB+ Diuretics +Nitrates & $6(2)$ \\
\hline CCB+ Diuretics +Diuretics & $3(1)$ \\
\hline Diuretics +ACE Inhibitor + Beta Blocker & $3(1)$ \\
\hline Diuretics +Alpha Beta Blocker+ ARBs & $3(1)$ \\
\hline Diuretics + Alpha Beta Blocker +Nitrates & $6(1)$ \\
\hline Diuretics +Diuretics +ARBs & $12(4)$ \\
\hline Diuretics +Beta Blocker +Alpha Agonist & $3(1)$ \\
\hline Diuretics +Diuretics + Beta Blocker & $3(1)$ \\
\hline Diuretics + Diuretics + ACE Inhibitor & $6(2)$ \\
\hline CCB+ACE Inhibitor +ACE Inhibitor & $9(1)$ \\
\hline Distribution of 4 \& 5 Drug Therapy & $9.09(1)$ \\
\hline CCB+ ACE Inhibitor + Alpha Beta Blocker + Beta \\
\hline Blocker
\end{tabular}




\section{WHO Prescribing indicators?}

The Drug utilization evaluation done by WHO prescribing indicators \{Table No.5\}.

\begin{tabular}{|l|l|l|}
\hline \multicolumn{2}{|l|}{ Table 5: Drug utilization in the study asper WHO } & Optimal range \\
\hline WHO Indicators & Encountered Values & $1.6-1.8$ \\
\hline Average no of drugs per patient with active drugs in FDCs & 1.825 & $100 \%$ \\
\hline Percentage of drugs prescribed by generic names & $34.50 \%$ & $13.4-24.1$ \\
\hline Percentage of encounters with injection prescribed & $11.87 \%$ & $100 \%$ \\
\hline Percentage of drugs prescribed from NLEM & $42.3 \%$ & \\
\hline
\end{tabular}

In the present study 95\% (n=190) of the prescriptions were following the JNC-8 guidelines for prescribing antihypertensive agents $\{$ Table No.6\}.

\begin{tabular}{|l|l|}
\hline Table 6: Distribution of Prescription based on Adherence to JNC-8. \\
\hline Adherence to JNC-8 & Percentage (n) \\
\hline Prescriptions adhering to JNC-8 & $95(190)$ \\
\hline Prescriptions not adhering to JNC-8 & $5(10)$ \\
\hline
\end{tabular}

In this study, the $9^{\text {th }}$ recommendation $(n=5)$ was found to be most violated among others $\{$ Fig. 2$\}$.

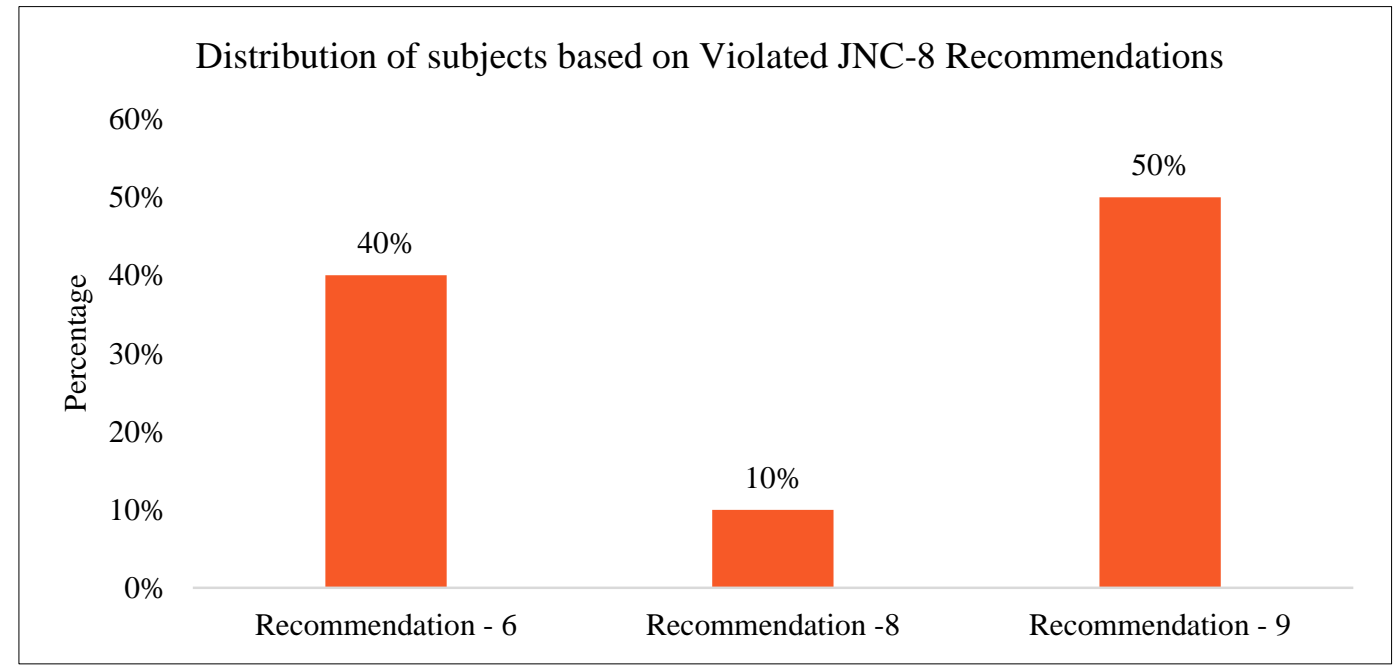

Figure 2: Distribution of subjects based on Violated JNC-8 Recommendations

\section{DRP analysis by PCNE}

By evaluating the PROBLEM domain by PCNE, Treatment safety (P2) related problems were the most common type $(74.52 \%)$ among others. By evaluating the CAUSES domain by PCNE classification, the most common cause for most of the DRP was found to be DRUG SELRCTION (C1) (63.06\%). By evaluating the PLANNED INTERVENTION domain based on PCNE classification, most of the DRPs were not having interventions (58\%). The remaining interventions are done mainly at patient level (22\%) and Prescriber level (19\%).

\section{DISCUSSION}

According to this study the average age of patients was found to be $69.7 \pm 7.66$ with female male ratio $27: 23$. Asper this study, hypertension is more prevalent in geriatric patients between the age group $65-70(74.00 \%)$. This study result is contrast with the study conducted by Romday $\mathbf{R}$ et al, because in their study 40-60 age groups was the most common age group ${ }^{1}$.
In the current study majority of the co morbidities were T2DM (36\%) followed by CNS diseases (15.0\%), CVS diseases $(13.70 \%)$. However, study conducted by Nayaka et al, had shown an inconsistent finding with the present study were majority of comorbidities were seen in RS diseases $66.67 \%$, followed by endocrine diseases $35.33 \%$, cardiovascular diseases $32.67 \%{ }^{2}$. This discrepancy in the outcome may be assumed due to the study subjects enrolled and comorbidities included. The study by Nayaka et al included all geriatric patients irrespective of their co- morbid diseases ${ }^{2}$.

The 8th report of JNC on detection, evaluation, prevention and treatment recommends the use of ACEIs, ARBs, Thiazide diuretics and CCBs alone or in combination for the management of early-stage hypertension. The findings of this study show that, out of 200 prescriptions, $53 \%$ of prescriptions contains combination therapy $(n=106)$ and remaining $47 \%(n=94)$ contains mono therapy. Similar study conducted by Sharma JK et al concluded that, monotherapy was prescribed in 48 . 59\% of total prescriptions and combination therapy was prescribed in $51.41 \%$ of total 
AHM Viswanatha Swamy et al

prescriptions $^{3}$. In the 106 combination therapies, most of the physicians prescribed 2 drug regimens (57.54\%). The most frequently prescribed class as mono therapy was Calcium channel blockers (CCB) 79. 00\% (n=74). The study conducted by Malpani et al and Siva Reddy et al concluded the same ${ }^{4,5}$. However this result was not compactable with the study conducted by Narkar et al where ARB was the most frequently used class ${ }^{6}$. Amlodipine was the only CCB repeatedly prescribed in this study $71.50 \% \quad(n=143)$. Similarly, Paulose et al summarized that amlodipine was the most frequently used antihypertensive as mono therapy ${ }^{7}$. The second most commonly prescribed class as mono therapy was BB $8.51 \%(n=8)$. Among BB, metoprolol was the commonest $8.50 \% \quad(n=17)$. These findings indicating disparities with study done by Sharma JK et al in small extent. In that BBs are the third most common class (5.48\%) and atenolol was the commonest prescribed drug3. In antihypertensive multi drug regimen, two drugs regimen was the commonest in this study $57.54 \%(n=61)$. These findings are in consonance with other study performed by Sharma JK et al, $38.43 \%$ were two drug regimen therapy ${ }^{3}$. Among two drug regimen CCB+ Diuretics combination are generally prescribed. Similarly, Pandaya et al. found that the most generally prescribed two-drug combination was CCB+ Diuretic (12 percent and $37.8 \%$, respectively) ${ }^{8}$. In this study, 32.07 percent $(n=34)$ of the participants were administered a three-drug antihypertensive medication regimen. CCBs + ARBs + Diuretic was the most usually recommended threedrug combination. This is consistent with the findings of Gupta et al, who found that three medication combinations as antihypertensives were most commonly used: CCBs+ ACEIs/ARBs+ Diuretic ${ }^{9}$. In this study, $10.37 \%$ of prescriptions contain $4 \& 5$ drug antihypertensive regimen. Generally prescribed 4 drug combination is CCB+ Alpha Beta blockers + Diuretic +ARBs $18 \%(n=2)$ and five drug combination is CCB+ Diuretic + Alpha Beta blockers +Diuretic + Alpha agonist $18 \%(\mathrm{n}=2)$. Overall CCBs are commonly prescribed antihypertensive drugs followed by diuretic. JNC 8 recommends use of CCBs, Diuretic; ACEI/ARBs are preferred drug classes both in mono therapy and combination therapy.

As per Prescribing indicators by World Health Organization (WHO), the average number of drugs per patient was found to be 1.825 , it was almost within in the optimal range (1.61.8). The percentage of encounters with injection was only $11.87 \%$, this value was below to the optimal range. The percentage of medications prescribed by their generic name was $34.5 \%$ and percentage of medications prescribed from the NLEM -2021 was $42.3 \%$. The both values suggesting that prescription writing using brand names were common and usage of drugs from NLEM was not optimal. Similar to the present study, WHO indicators for drug utilization was also used in the study conducted by Bhandary A et al, as per that average number of drugs per patient was 1.60 and the percentage of encounters with injection was only $2.21 \% 10$. The percentage of medications prescribed by generic names was $8.85 \%$ and percentage of medications prescribed from the essential drug list 2015 was only $32.30 \%$.

In this study overall rate of adherence to JNC 8 is $95 \%(n=190)$. Complete adherence rate was observed among the patients of Hypertensive urgency and emergency with the JNC 8 guideline. This result was supported by Gupta et al and similar conclusion also reported in study conducted by Ramday $\mathbf{R}$ et $\mathbf{a l}^{1}$. Remaining $5 \%(\mathrm{n}=10)$ prescriptions are not adhere to JNC 8. The least adherence is to recommendation $6,8,9$. According to JNC 8 , the recommendation 6 explain about first line antihypertensive agents (Thiazide diuretic, CCB, ARBs, ACEI). The recommendation 8 explain about treatment specifications for CKD patients. Their treatment
Journal of Drug Delivery \& Therapeutics. 2022; 12(1):72-77 should include an ACEI or ARBs to improve kidney outcome. The recommendation 9 gives idea about combination therapy. If BP is failed to reach in a required goal using first line drugs, then titrate the dose at maximum again if it is not achieving the goal then go for combination therapy of first lines after that second line drugs.

In this study, a total of 157 DRPs are identified. By evaluating Problems based on PCNE, the most commonly encountered problems are treatment safety related problems $74 \%$ (n= 117). By assessing Causes as per PCNE, regarding drug choice problem, the results agree with studies conducted by Redzuan $\mathbf{A M}$ et $\mathbf{a l}^{11}$. The current study indicating that, around $63 \%(n=99)$ of DRPs are associated with drug selection. The high frequency of drug selection issues indicated that pharmaceuticals should be prescribed with greater caution, particularly in elderly patients. By analysing planned interventions, most of the cases have no interventions. The majority of interventions accomplished at patient $22.00 \%(\mathrm{n}=35)$ and prescriber $19.00 \%(\mathrm{n}=29)$ level.

This study conducted was single centred and carried out only in inpatient department, therefore population was relatively homogenous. The sample size, which is included in the study was less. Due to this variableness and ambiguity should be considered as limitations. Despite these limitations the study revealed various significant features of the Drug utilization patterns and adherence of antihypertensive drugs to JNC 8 guidelines in diverse co-morbid conditions.

\section{CONCLUSION}

In this study by analysing prescription pattern, we concluded that most commonly prescribed drug class was CCB (Amlodipine) followed by BB (Metoprolol). By analysing prescribing indicators by $\mathrm{WHO}$ revealed that, prescription writing using generic name and usage of drugs from NLEM need to be encouraged. Present study results demonstrating that, most of the physicians are adherent to the JNC 8 recommendations. But provider adherence in recommendations 6 and recommendation 9 has room for improvement. A regular monitoring of the prescription pattern and a new strategy of provider educational methods need to be promoted to keep a tab on the rational drug prescriptions for a better therapeutic outcome. Inclusion of clinical/hospital pharmacist in drug therapy monitoring and prescription analysis should enhance. This study disclosing that, treatment safety related problems were the most commonly observed DRP as per PCNE classification. By considering this, more interventions are essential to ensure the treatment safety and effectiveness in study population. In the current study population majority of them are illiterate and under below poverty level. Due to their illiteracy and financial instability, they are not adhering to the medication and they are at higher risk of morbidity and mortality.

\section{ACKNOWLEDGEMENT}

We are over helmed in all humbleness and gratefulness to acknowledge our depth to guides and other faculty members who directly or indirectly lend a helping hand to accomplish this research.

\section{CONFLICTS OF INTEREST}

The authors conform that there are no conflicts of interest.

\section{ABRIVATIONS}

ACE: Angiotensin Converting Enzyme ;ARB: Angiotensin Receptor Blocker ;BB: Beta Blocker ;CCB: Calcium Channel Blocker ;CHF: Congestive Heart Failure ;CKD: Chronic Kidney Disease ;CNS: Central Nervous System ;CVS: Cardiovascular System ;DRP: Drug Related Problems ;FDC: Fixed Dose 


\section{AHM Viswanatha Swamy et al}

Combination ;JNC: Joint National Committee ;NLEM

National List of Essential Medicine ;PCNE: Pharmaceutical

Care Network of Europe ;T2DM: Type 2 Diabetes mellitus

;WHO: World Health Organization.

\section{REFERENCES}

1) Romday R, Gupta A, Bhambani P. An assessment of antihypertensive drug prescription patterns and adherence to joint national committee-8 hypertension treatment guidelines among hypertensive patients attending a tertiary care teaching hospital. Int J Res Med Sci. 2016; 5125-33. https://doi.org/10.18203/2320-6012.ijrms20164046

2) Nayaka S, Rajeshwari B, Venkatadri T. Drug utilization pattern in geriatric inpatients of medicine department in a Tertiary Care Teaching Hospital. Int J Basic Clin Pharmacol. 2015; 568-73. https://doi.org/10.18203/2319-2003.ijbcp20150042

3) Sharma JK, Parmar SP, Trivedi HR. A study of prescribing pattern of antihypertensive drugs in hypertensive patients with co morbid diabetes in a tertiary care teaching hospital. Int J Basic Clin Pharmacol. 2018; 7(3):375. https://doi.org/10.18203/2319-2003.ijbcp20180486

4) Malpani AK, Waggi M, Panja P, Christien TM. Study of prescribing pattern of antihypertensive drugs and evaluation of the prescription with JNC 8 guidelines in north karnataka hospital. Ind J Pharm Pr. 2018; 11(4):193-7. https://doi.org/10.5530/ijopp.11.4.40

5) Pingili, RB. An Assessment of Antihypertensive Drug Utilization Patterns and Adherence to JNC-7 Guidelines in South Indian Tertiary Care Teaching Hospital. Ind J Pharm Pr.2015; 8(4):177182.
Journal of Drug Delivery \& Therapeutics. 2022; 12(1):72-77

6) Narkar NS, Deshpande T, Rane BT, Kothari R, Tilak AV, Bhide H. Pattern of antihypertensive drugs prescribed in a tertiary care hospital in western India. Biomed Pharmacol J. 2021; 14(02):961-9. https://doi.org/10.13005/bpj/2197

7) Bairy LK. Comparison of efficacy and adverse drug reactions of monotherapy versus combination therapy of antihypertensives among diabetic hypertensive patients in a tertiary care hospital. Asian J Pharm Clin Res. 2017; 10(2):385. https://doi.org/10.22159/ajpcr.2017.v10i2.15854

8) Pandaya S, Ferdous MR ul, Panthi VK, Pandey M, Maharjan L. Effectiveness of antihypertensive agents among hypertensive patients of Nepal. Int Res J Pharm. 2014; 5(12):903-9. https://doi.org/10.7897/2230-8407.0512183

9) Gupta CN, Sk A, Mahapatra S, Lahiri A, Maji K, Sen S. Evaluation of antihypertensive drug prescription patterns, rationality, and adherence to joint national committee-8 hypertension treatment guidelines among patients attending medicine OPD in a tertiary care hospital. Int J Contemp Med Res [IJCMR][Internet]. 2019; 6(10). https://doi.org/10.21276/ijcmr.2019.6.10.42

10) Bhandary A, R. Bhandari P. Drug usage pattern of antihypertensive drugs in elderly diabetic, hypertensive in-patients with or without impaired renal function in a tertiary care hospital. Int J Basic Clin Pharmacol. 2018; 7(4):696. https://doi.org/10.18203/2319-2003.ijbcp20181172

11) Redzuan AM. Drug-Related Problems in Hypertensive Patients with Comorbidities. J Pharm Res. 2017; 1(3):000113. https://doi.org/10.23880/OAJPR-16000113 